\title{
Uniqueness of Products in Higher Algebraic $\mathbb{K}$-Theory
}

Dedicated to Professor Hirosi Toda on his 60th birthday

By

\author{
Kazuhisa SHIMAKAWA*
}

\section{Introduction}

Let $\mathbb{E}$ be a higher algebraic $\mathrm{K}$-theory defined on rings, that is, a functor which assigns to each ring $R$ a spectrum $\mathbb{E} R$ of algebraic $\mathrm{K}$ theory of $R$. Fiedorowicz uniqueness theorem [2] says that if has an external tensor product, then there is a natural map of spectra

$$
f: \mathbb{E} R \rightarrow \mathbb{G} \mathbb{W} R
$$

which induces an equivalence between $(-1)$-connected covers of $\mathbb{E} R$ and the Gersten-Wagoner spectrum $G \mathbb{W} R$ ([3] and [13]). May [6] has given a similar uniquenes theorem for higher algebraic $\mathrm{K}$ theories (or, infinite loop space machines) defined on permutative (i. e., symmetric strict monoidal) categories: given an infinite loop space machine $\mathbb{E}$ defined on permutative categories, there exists a natural equivalence of spectra between $\mathbb{E} U$ and the spectrum $\mathbb{S} B \bar{U}$ constructed by Segal [9].

In the present article we study the multiplicativity of such natural transformations between higher algebraic $\mathrm{K}$-theories defined on permutative categories, or exact categories, or rings. Here the term 'multiplicativity' is used in the following sense。 Let $\mathbb{E}$ and $\mathbb{S}^{\prime}$ ' be functors $\mathscr{C} \rightarrow \mathscr{S}$ from permutative categories (or exact categories, or rings) to $C W$-spectra, and suppose that $\mathbb{E}^{\prime}$ (resp. functorially associates to each pairing $U \times V \rightarrow W$ in $\mathscr{C}$ a pairing $\mathbb{E} U \wedge \mathbb{E} V \rightarrow \mathbb{E} W$ (resp. $\mathbb{E}^{\prime} U \wedge \mathbb{E}^{\prime} V \rightarrow \mathbb{E}^{\prime} W$ ) of $C W$-spectra. Then a natural transformation $f: \mathbb{E} \rightarrow \mathbb{E}^{\prime}$ is called multiplicative if the following square commutes

Received April 27, 1987.

* Research Institute for Mathematical Sciences, Kyoto University, Kyoto 606, Japan. 
in the homotopy category $H \mathscr{S}$;

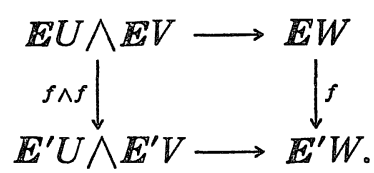

Notice that most of the constructions of products in higher algebraic $\mathrm{K}$-theory, except for May's [7], provide only weak pairings, i. e., pairings in the sense of G.W. Whitehead. This notion of a weak pairing is inadequate for sophisticated spectrum level analysis. Hence we want to find a condition, as generous as possible, which ensures that a given machine functorially associates 'true' pairings. Thus we introduce a notion of a pairing of $S_{*}$-spectra which gener-

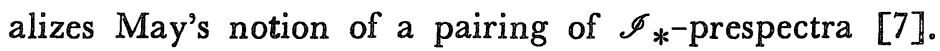

We now state the results of the paper.

A $C W$-spectrum $E=\left\{E_{n} \mid n \geqq 0\right\}$ is called an $S_{*}$-spectrum if each $E_{n}$ has an action by the symmetric group $S_{n}$ ( $E_{n}$ is an $S_{n}-C W$ complex) which is compatible with the structure maps and restricts to a homotopically trivial $A_{n}$-action. (See Section 1.) There is a relevant notion of a pairing of $S_{*}$-spectra and we can show that pairings $(E, F) \rightarrow G$ of $S_{*}$-spectra functorially determine pairings $E \wedge F \rightarrow G$ in the stable category。

We use the term higher algebraic $\mathrm{K}$-theory defined on permutative categories to denote a functor $\mathbb{E}$ which assigns to every permutative category $U$ a connective $C W$-spectrum $U=\left\{\mathbb{E}_{n} U \mid n \geqq 0\right\}$ together with a natural map $\lambda: B U \rightarrow \mathbb{E}_{0} U$ such that the composite $B U \rightarrow \Omega^{\infty} \mathbb{E}_{\infty} U=$ $\cup_{n} \Omega^{n} \mathbb{E}_{n} U$ is a group completion.

Definition. A higher algebraic $\mathrm{K}$-theory $\mathbb{E}$ defined on permutative categories is called multiplicative if (i) $\mathbb{E} U$ has a natural structure of an $S_{*}$-spectrum, and (ii) given a pairing $f: U \times V \rightarrow W$ of permutative categories, there exists a natural pairing $\mathbb{E} f=\left\{\mathbb{E}_{m, n} f\right\}:(\mathbb{E} U, \mathbb{E} V) \rightarrow \mathbb{E} W$ of $S_{*}$-spectra such that the following square commutes;

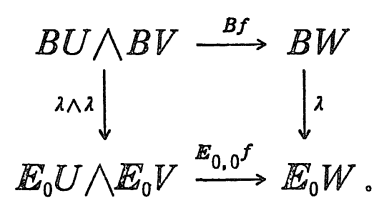

Thus a multiplicative higher algebraic $\mathrm{K}$-theory $\mathbb{E}$ functorially 
associates a true pairing $\mathbb{E} f: \mathbb{E} V \rightarrow W$ of $C W$-spectra.

It will be shown that both May machine $1 / \mathbb{R}_{\text {[7] }}$ and ShimadaShimakawa machine $C[10]$ are multiplicative higher algebraic $\mathbb{K}$ theories defined on permutative categories. (But Segal's machine [9] is not.)

Now our first theorem is

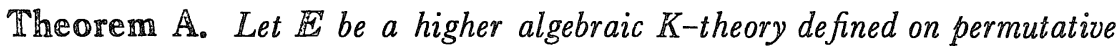
categories. Then there is a natural equivalence $\gamma: \mathbb{E} \rightarrow \mathbb{C} U$ which is multiplicative when is a multiplicative higher algebraic $K$-iheory.

Note. Because the passage from symmetric monoidal to permutative categories preserves pairings (cf. $[7, \S 2]$ ), every multiplicative higher algebraic $\mathbb{K}$-theory defined on permutative categories (e。g. MI) can be canonically regarded as a multiplicative higher algebraic $\mathbb{K}$-theory defined on symmetric monoidal categories. (We omit the obvious definition of the latter notion.) Theorem $A$ holds true for any $\mathbb{E}$ defined on symmetric monoidal categories.

Next let $\mathbb{K}$ denote the Waldhausen machine [14] which assigns to each exact category $U$ a $C W$-spectrum $\mathbb{K} U=\left\{B Q^{n} U^{[n]} \mid n \geqq 0\right\}$ (cf. [11]). Then $K$ associates to any biexact functor $f: U \times V \rightarrow W$ a pairing $\mathbb{K} f:(\mathbb{K} U, \mathbb{K} V) \rightarrow \mathbb{K} W$ of $S_{*}$-spectra. (This is essentially the result of [11].) Let us denote by Is $U$ the subcategory of all isomorphisms in a category $U$, and consider both $\mathbb{I s} U$ and $Q U$ as symmetric monoidal categories.

Theorem $\mathbb{B}$. There is a multiplicative natural transformation $\nVdash: \mathbb{C} \mathbb{S} U$ $\rightarrow \mathbb{K} U$ defined as the composite of a natural equivalence $\eta: \Omega C Q U \cong \mathbb{K} U$ with a natural map $\nu: \mathbb{C I s} U \rightarrow \Omega \mathbb{C Q U}$ which deloops the familiar map $B I s U \rightarrow \Omega B Q U$ 。

Note that by the " $+=Q "$ theorem $[4], \kappa$ becomes an equivalence if every short exact sequence in $U$ splits.

Finally we consider higher algebraic $\mathbb{K}$-theories defined on rings. We do not know whether Loday's pairing $\left(G \mathbb{W} R, G \mathbb{G} R^{\prime}\right) \rightarrow \mathbb{G} \mathbb{W}\left(R \otimes R^{\prime}\right)$ induces a 'true' pairing $\mathbb{G} W R \wedge \mathbb{G}\left[\mathbb{W}^{\prime} \rightarrow \mathbb{G} \mathbb{W}^{\prime}\left(R \otimes R^{\prime}\right)\right.$ or not. However we have 
Theorem C. There exists a functor $\boldsymbol{A}$ from rings to $S_{*^{-}}$spectra which satisfies the followings:

(1) There is a natural pairing $\mu:\left(A R, A R^{\prime}\right) \rightarrow A\left(R \otimes R^{\prime}\right)$ of $S_{*^{-}}$ spectra.

(2) For each $n \geqq 1$, there is a natural group completion $f_{n}: B I s P\left(S^{n} R\right) \rightarrow$ $A_{n} R\left(\simeq K_{0} S^{n} R \times B G L S^{n} R^{+}=G W_{n} R\right)$ such that

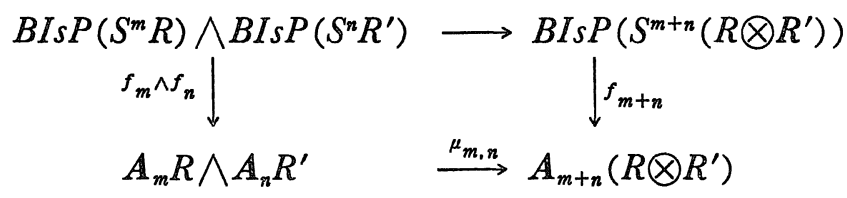

commutes. (Here $P(R)$ denotes the category of finitely generated projective modules over $R_{0}$ )

(3) The structure map $A_{n} R \wedge S^{1} \rightarrow A_{n+1} R$ is given by the composite

$$
A_{n} R \wedge S^{1} \stackrel{1 \wedge \iota}{\longrightarrow} A_{n} R \wedge A_{1} Z \stackrel{\mu_{n, 1}}{\longrightarrow} A_{n+1}(R \otimes Z)=A_{n+1} R
$$

where $\iota: S^{1} \rightarrow A_{1} \mathbb{Z}$ represents the standard generator of $K_{1} S \mathbb{Z}=\mathbb{Z}$ (cf. [5, Chapitre $I I]$ ).

(4) There is a multiplicative natural transformation $\alpha: C I s P(R) \rightarrow A R$ such that the induced map $\Omega^{\infty} C_{\infty} I s \mathrm{P}(R) \rightarrow \Omega^{\infty} A_{\infty} R$ is an equivalence.

Note that the condition (3) is similar to the description of the structure map of $G W R$ given by Loday [5]. From (2) we see that $\mu_{m, n}$ is weakly homotopic to Loday's map $G W_{m} R \wedge G W_{n} R^{\prime} \rightarrow G W_{m, n}\left(R \otimes R^{\prime}\right)$.

As a consequence we have

Corollary. (Cf. Weibel [15].) The product structures in higher algebraic $K$-theory of rings constructed by Waldhausen [14], May [7], ShimadaShimakawa [10], and Loday [5] (modified as in Theorem G) all agree with each other.

The proofs of the above theorems are given in the final section. In Section 1 we introduce a notion of a pairing of $S_{*}$-spectra and prove that pairings of $S_{*}$-spectra functorially determine pairings in the stable category. Section 2 illustrates how the machines of Waldhausen, Shimada-Shimakawa, and May associate pairings of 
$S_{*}$-spectra, and Section 3 provides a key tool on which our proofs of the theorems are based, that is to say, a multiplicative version of the 'up and across theorem' $[2,8]$.

\section{§1. Pairings of $\mathbb{S}_{*}$-Spectra}

Throughout the paper we regard $S^{1}$ as the one-point compactification of $R\left(\{\infty\}\right.$ is the base-point), and denote by $S^{n}$ the smash product of $n$ copies of $S^{1}$. Each $S^{n}$ is an $S_{n}-C W$ complex equipped with the standard $S_{n}$-action; $\sigma\left(s_{1}, \ldots, s_{n}\right)=\left(s_{0^{-1}(1)}, \ldots, s_{\sigma^{-1}(n)}\right)$ for every permutation $\sigma \in S_{n}$ 。

Let $E=\left\{E_{n} \mid n \geqq 0\right\}$ be a $C W$-spectrum. We say that $E$ is an $S_{*^{-}}$ spectrum if each $E_{n}$ is a based $S_{n}-C W$ complex, and if the following two conditions hold: (i) the diagram

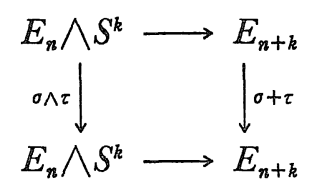

commutes for all $\sigma \in S_{n}$ and $\tau \in S_{k}$; and (ii) for every even permutation $\sigma \in A_{n}$ the map $\sigma: E_{n} \rightarrow E_{n}$ is homotopic to the identity. Given $S_{*}$-spectra $E$ and $F$, a function $f: E \rightarrow F$ is called a function of $S_{*}-$ spectra if each $f_{n}: E_{n} \rightarrow F_{n}$ is $S_{n}$-equivariant. A map of $S_{*}$-spectra is a map $f: E \rightarrow F$ which is represented by a function $f^{\prime}: E^{\prime} \rightarrow F$ of $S_{*}$-spectra for some cofinal subspectrum $E^{\prime}$ such that each $E_{n}^{\prime}$ is invariant under the $S_{n}$ action on $E_{n}$.

If we consider $S_{*}=\amalg_{n} S_{n}$ as the skeletal category of finite sets and their isomorphims, then each $S_{*}$-spectrum $E$ can be regarded as a functor from $S_{*}$ to $C W$-complexes. Moreover the structure maps $E_{m} \wedge S^{n} \rightarrow E_{m+n}$ constitute a natural transformation $E \wedge S^{0} \longrightarrow E_{\circ} \oplus$ where $S^{0}: \mathfrak{n} \mapsto S^{n}$ denotes the sphere-valued functor. It follows that an $\mathscr{I}_{*^{-}}$ prespectrum in the sense of May [7] restricts, via the canonical embedding $S_{*} \rightarrow \mathscr{I}_{*}$, to an $S_{*}$-spectrum in our sense. (Strictly speaking, $\mathscr{I}_{*}$-prespectra are not supposed to have a structure of a $C W$-spectrum. But this is not serious because the passage from $\mathscr{I}_{*}$-prespectra to the stable category is equivalent to the process of replacing spectra by $C W$-approximations.) 
Note that in the definition of an $S_{*}$-spectrum the condition (ii) follows from (i) if the $S_{n}$-action on $E_{n}$ extends to an $O(n)$-action (e. g., $E$ is an $\mathscr{I}_{*}$-prespectrum), or if $E$ is an almost $\Omega$-spectrum, that is, the maps $E_{n} \rightarrow \Omega E_{n+1}$ are homotopy equivalences for $n \geqq 1$ (cf. [11, Lemma 4.1]).

Now let $E, F$ and $G$ be $S_{*}$-spectra。

Definition 1.1. A pairing of $S_{*}$-spectra $\mu:(E, F) \rightarrow G$ is a family of maps

$$
\mu_{m, n}: E_{m} \wedge F_{n} \longrightarrow G_{m+n} ; \quad m, n \geqq 0
$$

such that the following diagram commutes;

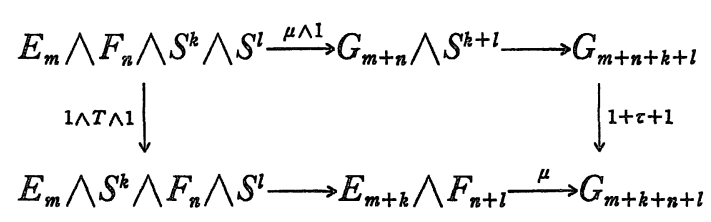

where $1+\tau+1$ denotes the permutation

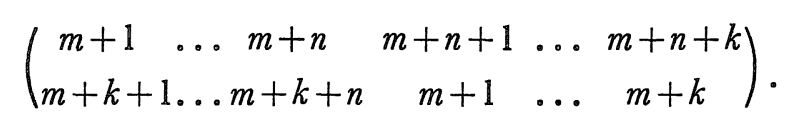

Example 1.2. Let $E$ be an $S_{*}$-spectrum. Then the canonical pairing $\varepsilon:\left(E, S^{0}\right) \rightarrow E$ consisting of the maps $E_{m} \wedge S^{n} \rightarrow E_{m+n}$ is a pairing of $S_{*}$-spectra.

We now describe the passage from pairings of $S_{*}$-spectra to pairings in the stable category $H \mathscr{S}$.

Given an $S_{*}$-spectrum $G$ we construct a sort of double telescope $W G$ as follows. For every $n \geqq 0, W G_{n}$ is defined as an identification space of the union

$$
\begin{aligned}
& \bigvee_{i+j \leq n}([i] \times[j])+\wedge G_{i+j} \wedge S^{n-i-j} \\
& \bigvee \bigvee_{i+j \leq n-1}([i] \times[j, j+1])+\wedge G_{i+j} \wedge S^{n-i-j} \\
& \bigvee \bigvee_{i+j \leq n-1}([i, \quad i+1] \times[j])+\wedge G_{i+j} \wedge S^{n-i-j} \\
& \bigvee \bigvee_{i+j \leq n-2} G_{i+j} \wedge M(\tau) \wedge S^{n-i-j-2}
\end{aligned}
$$

where $M(\tau)$ denotes the Thom space of a certain $S O(2)$-bundle $\tau$ over the 2 -cell $[i, i+1] \times[j, j+1]$ and we identify 


$$
\begin{aligned}
((i, j), g, s) & \in([i] \times[j, j+1])+\wedge G_{i+j} \wedge S^{n-i-j} \quad \text { or } \\
& \in([i, i+1] \times[j])+\wedge G_{i+j} \wedge S^{n-i-j}
\end{aligned}
$$

with

$$
\begin{aligned}
& ((i, j), g, s) \in([i] \times[j])+\wedge G_{i+j} \wedge S^{n-i-j} \\
& ((i, j+1), g, s, t) \in([i] \times[j, j+1])+\wedge G_{i+j} \wedge S^{1} \wedge S^{n-i-j-1}
\end{aligned}
$$

with

$$
((i, j+1),[g, s], t) \in([i] \times[j+1])+\wedge G_{i+j+1} \wedge S^{n-i-j-1}
$$

and

$$
((i+1, j), g, s, t) \in([i,+1] \times[j])+\wedge G_{i+j} \wedge S^{1} \wedge S^{n-i-j-1}
$$

with

$$
\left((i+1, j), \sigma_{i, j}\left[g,(-1)^{j} s\right], t\right) \in([i+1] \times[j])+\wedge G_{i+1+j} \wedge S^{n-i-1-j}
$$

where

$$
\sigma_{i, j}=\left(\begin{array}{lll}
i+1 & i+2 \cdots i+j+1 \\
i+2 & i+3 \cdots & \cdots+1
\end{array}\right) \in S_{i+j+1} 。
$$

(The identification of $G_{i+j} \wedge M(\tau) \wedge S^{n-i-j-2}$ with the part already constructed is quite similar to that described in [1, p. 175].) The structure maps are obvious. (Compare with the definition of the smash product of spectra $[1, \S 4]$.)

If $\mu:(E, F) \rightarrow G$ is a pairing of $S_{*}$-spectra, then we have

$$
\mu_{i+1, j}\left(\left[e,(-1)^{j} s\right], f\right)=\sigma_{i, j}\left[\mu_{i, j}(e, f),(-1)^{j} s\right]
$$

for all $(e, f, s) \in E_{i} \wedge F_{j} \wedge S^{1}$. Hence there is a well-defined map

$$
\bar{\mu}: \mathbb{E} \wedge F \rightarrow W G \text {. }
$$

Lemma 1.3. Pairings $\mu:(E, F) \rightarrow G$ of $S_{*}$-spectra functorially determine pairings

$$
\tilde{\mu}: E \wedge F \rightarrow G
$$

in the stable category $H \mathscr{S}$.

Proof. There is a sequence of natural maps of spectra

$$
E \wedge F \stackrel{\bar{\mu}}{\longrightarrow} W G \stackrel{\bar{\varepsilon}}{\longrightarrow} G \wedge S^{0} \stackrel{\simeq}{\longleftarrow} T G \stackrel{\simeq}{\longrightarrow} G
$$

where $T G$ denotes the telescope of $G$. To define $\tilde{\mu}$ we have only to prove that $\bar{\varepsilon}: G \wedge S^{0} \rightarrow W G$ is a homotopy equivalence. 
Take a partition $A=B \cup C$ of an ordered set $A \cong N$, and define a spectrum $W_{B C} G$ as follows: for every $a \in A$ we put

$$
W_{B C} G_{\alpha(a)}=G_{\beta(a)+\gamma(a)},
$$

where $\alpha(a)=\#\{x \in A \mid x<a\}$ etc., and identify $(g, s) \in W_{B C} G_{\alpha(a)} \wedge S^{1}$ with

$$
\begin{array}{ll}
{[g, s] \in G_{\beta(a)+(\gamma(a)+1)}} & \text { if } a \in C \\
\sigma_{B(a), \gamma(a)}\left[g,(-1)^{r^{(a)} s}\right] \in G_{(\beta(a)+1)+\gamma(a)} & \text { if } a \in B .
\end{array}
$$

(Compare with the definition of naive smash product [1].) Now suppose that both $B$ and $C$ are infinite and that $\gamma(a)$ is even (and hence $\sigma_{\beta(a), \gamma(a)} \simeq \mathrm{id)}$ whenever $a \in B$. Then we have a commutative diagram

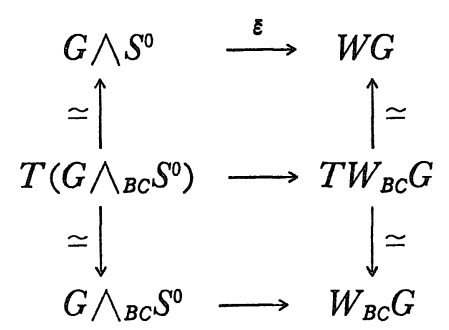

in which every vertical map is a homotopy equivalence. Since $W_{B C} G_{n} \wedge S^{1} \rightarrow W_{B C} G_{n+1}$ are homotopic to the original structure maps $G_{n} \wedge S^{1} \rightarrow G_{n+1}$, the bottom map becomes a weak homotopy equivalence. Thus we see that $\bar{\varepsilon}$ is a homotopy equivalence.

Notation. In what follows we use the same letter $\mu$ to denote the pairing $E \wedge F \rightarrow G$ induced from a pairing $\mu:(E, F) \rightarrow G$ of $S_{*}$-spectra.

\section{§ 2. Multiplicative Higher Algebraic K-Theories}

2.1. Waldhausen machine. For every exact category $U$ we have a $C W$-spectrum $\boldsymbol{K} U=\left\{\boldsymbol{K}_{n} U \mid n \geqq 0\right\}$ where

$$
\boldsymbol{K}_{n} U=B Q^{n} U^{[n]}=B Q_{1} \cdots Q_{n} U^{[n]}
$$

denotes the classifying space of the $n$-fold category obtained by applying $Q$ on every component of the $n$-fold exact category $U^{[n]}$ of commutative $n$-cubes in $U$ (cf. [11]). Note that $\boldsymbol{K}_{0} U$ is the set $o U$ of all objects of $U$. (This differs from the definition given in [11] in which $\boldsymbol{K}_{0} U$ is defined to be $\Omega B Q U$.)

We have shown in [11] that the evident $S_{n}$-action on $U^{[n]}$ induces 
an $S_{n}$-action on $B Q^{n} U^{[n]}$ with respect to which $\mathbb{K} U$ becomes an $S_{*^{-}}$ spectrum. Moreover any biexact functor $f: U \times V \rightarrow W$ induces a natural map

$$
\mathbb{K}_{m, n} f: B Q^{m} U^{[m]} \wedge B Q^{n} V^{[n]} \rightarrow B Q^{m+n} W^{[m+n]}
$$

for each pair of integers $m$ and $n$. The diagram (4.2) in [11] shows that $\mathbb{K}_{m, n} f$ define a pairing $\mathbb{K} f:(\mathbb{K} U, \mathbb{K} V) \rightarrow \mathbb{K} W$ of $S_{*}$-spectra. Thus $f$ functorially associates a pairing

$$
\mathbb{K} f: \mathbb{K} U \wedge \mathbb{K} V \rightarrow \mathbb{K} W
$$

in the stable category.

2.2. Shimada-Shimakawa machine. In [10] we have associated to any symmetric monoidal category $U=\langle U, \oplus\rangle$ a spectrum $\mathbb{C} U=$ $\left\{B \mathscr{B}{ }^{n} U \mid n \geqq 0\right\}$ where $\mathscr{B}$ is a functor which assigns to each symmetric monoidal (topological) category $C$ a symmetric monoidal category $\mathscr{B} C$ together with a natural map $B C \wedge S^{1} \rightarrow B \mathscr{B} C$. By extending the arguement of $\left[10\right.$, Lemma 2.6] we see that $\mathbb{C}_{n} U=B \mathscr{B}^{n} U$ is identical with the geometric realization of a $\Gamma^{n}$-space $B \hat{B}^{n} U$ defined as follows.

For each $\left(\mathbb{r}_{1}, \ldots, \mathbb{r}_{n}\right) \in \Gamma^{n}$, denote by $\hat{B}^{n} U\left(\mathbb{r}_{1}, \ldots, \mathbb{F}_{n}\right)$ the symmetric monoidal category with objects

$$
\left\langle a ; \alpha^{1}, \ldots, \alpha^{n}\right\rangle
$$

where $a$ is a function which assigns to each $n$-tuple $\left(T_{1}, \ldots, T_{n}\right)$ of subsets $T_{i} \subset \mathrm{r}_{i}$ an object $a\left(T_{1}, \ldots, T_{n}\right)$ of $U, \alpha^{i}$ is a family of isomorphisms

$a\left(T_{1}, \ldots, T_{i} \amalg T_{i}^{\prime}, \ldots, T_{n}\right) \stackrel{\cong}{\longrightarrow}\left(T_{1}, \ldots, T_{i}, \ldots, T_{n}\right) \oplus a\left(T_{1}, \ldots, T_{i}^{\prime}, \ldots, T_{n}\right)$

satisfying the conditions similar to those of [10, Definition 2.1 (i) ], and for any $T$ of the form $\left(T_{1}, \ldots, T_{i}^{0} \amalg T_{i}^{1}, \ldots, T_{j}^{0} \amalg T_{j}^{1}, \ldots, T_{n}\right)$ the following diagram commutes;

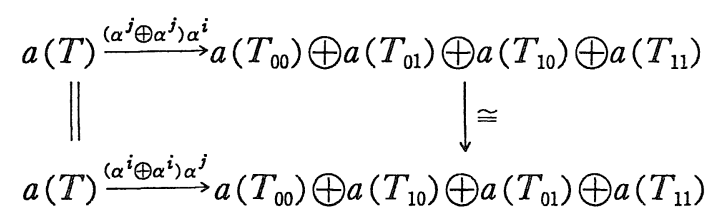

in which $T_{\varepsilon \delta}=\left(T_{1}, \ldots, T_{i}^{\varepsilon}, \ldots, T_{j}^{\delta}, \ldots, T_{n}\right) \quad(0 \leqq \varepsilon, \delta \leqq 1)$. Given objects $\left\langle a ; \alpha^{1}, \ldots, \alpha^{n}\right\rangle$ and $\left\langle b ; \beta^{1}, \ldots, \beta^{n}\right\rangle$ a morphism $f:\left\langle a ; \alpha^{1}, \ldots, \alpha^{n}\right\rangle \rightarrow\left\langle b ; \beta^{1}\right.$, $\left.\ldots, \beta^{n}\right\rangle$ is a family of morphisms $f\left(T_{1}, \ldots, T_{n}\right): a\left(T_{1}, \ldots, T_{n}\right) \rightarrow b\left(T_{1}\right.$, 
$\ldots, T_{n}$ ) compatible with all $\alpha^{i}$ and $\beta^{i}$ in the sense of $[10$, Definition 2. 1]. (Note that $\hat{B} U=\hat{B}^{1} U$ coincides with $\bar{U}$ of May [6].)

There is a canonical isomorphism

$$
\hat{B}^{n+1} U\left(\mathbf{r}_{1}, \ldots, \mathbf{r}_{n+1}\right) \cong \hat{B}\left(\hat{B}^{n} U\left(\mathbf{r}_{1}, \ldots, \mathbf{r}_{n}\right)\right)\left(\mathbf{r}_{n+1}\right)
$$

natural in both $U$ and $\left(\mathbf{r}_{1}, \ldots, \mathbf{r}_{n+1}\right)$. Hence, as in [10, §2], we can inductively prove that $C_{n} U=B \mathscr{B}^{n} U$ is isomorphic to the geometric realization of the $\Gamma^{n}$-space $B \hat{B}^{n} U$. Moreover the structure map $C_{n} U \wedge S^{1} \rightarrow C_{n+1} U$ is described as the inclusion

$$
\left|B \hat{B}^{n} U\right| \wedge S^{1} \cong\left|B \hat{B}^{n+1} U(\cdots, 1)\right| \wedge S^{1} \rightarrow\left|B \hat{B}^{n+1} U\right| .
$$

We now define an $S_{n}$-action on $C_{n} U$. Given a $\Gamma^{n}$-category $E$ and $\sigma \in S_{n}$, denote by $E^{\sigma}$ the $\Gamma^{n}$-category such that $E^{\sigma}\left(\mathbf{r}_{1}, \ldots, \mathbf{r}_{n}\right)=$ $E\left(\mathbf{r}_{\sigma^{-1}(1)}, \ldots, \mathbf{r}_{\sigma^{-1}(n)}\right)$. Clearly we have $\left|B E^{o}\right|=|B E|$ (cf. $[11,1.14]$ ). Returning to our case, for every $\sigma \in S_{n}$ there is a natural isomorphism $\Theta(\sigma): \hat{B}^{n} U \rightarrow\left(\hat{B}^{n} U\right)^{\sigma}$ which assigns to each $\boldsymbol{a}=\left\langle a ; \alpha^{1}, \ldots, \alpha^{n}\right\rangle \in \hat{B}^{n} U\left(\mathbf{r}_{1}\right.$, $\left.\ldots, \mathbf{r}_{n}\right)$,

$$
\Theta(\sigma) \boldsymbol{a}=\left\langle\tilde{a}: \tilde{\alpha}^{1}, \ldots, \tilde{\alpha}^{n}\right\rangle \in \hat{B}^{n} U\left(\mathbf{r}_{\sigma^{-1}(1)}, \ldots, \mathbf{r}_{\sigma^{-1}(n)}\right)
$$

where $\tilde{a}\left(T_{\sigma^{-1}(1)}, \ldots, T_{\sigma^{-1}(n)}\right)=a\left(T_{1}, \ldots, T_{n}\right)$ and $\tilde{\alpha}^{\sigma^{-1}(i)}=\alpha^{i}, 1 \leqq i \leqq n$. It is easy to see that the induced maps

$$
\begin{aligned}
& \left|B \hat{B}^{n} U\right| \stackrel{|B \theta(\sigma)|}{\longrightarrow}\left|B\left(\hat{B}^{n} U\right)^{\sigma}\right|=\left|B \hat{B}^{n} U\right| \\
& \Psi \\
& {\left[\boldsymbol{a}, s_{1}, \ldots, s_{n}\right] \longmapsto\left[\Theta(\sigma) \boldsymbol{a}, s_{\sigma^{-1}(1)}, \ldots, s_{\sigma^{-1}(n)}\right]}
\end{aligned}
$$

define an $S_{n}$-action on $C_{n} U$ with respect to which $C U$ becomes an $S_{*}$-spectrum.

Let $f: U \times V \rightarrow W$ be a pairing of symmetric monoidal categories. Then we define a map

$$
f_{m, n}: \hat{B}^{m} U\left(\mathbf{r}_{1}, \ldots, \mathbf{r}_{m}\right) \times \hat{B}^{n} V\left(\mathbf{r}_{m+1}, \ldots, \mathbf{r}_{m+n}\right) \rightarrow \hat{B}^{m+n} W\left(\mathbf{r}_{1}, \ldots, \mathbf{r}_{m+n}\right)
$$

of $\Gamma^{m+n}$-categories by

$$
\begin{aligned}
& f_{m, n}\left(\left\langle a ; \alpha^{1}, \ldots, \alpha^{m}\right\rangle,\left\langle b ; \beta^{1}, \ldots, \beta^{n}\right\rangle\right)= \\
& \quad\left\langle f(a \times b) ; \delta\left(\alpha^{1} \times 1\right), \ldots, \delta\left(\alpha^{m} \times 1\right), \delta^{\prime}\left(1 \times \beta^{1}\right), \ldots, \delta^{\prime}\left(1 \times \beta^{n}\right)\right\rangle
\end{aligned}
$$

where $\delta\left(\alpha^{i} \times 1\right)$ denotes the family of composite isomorphisms

$$
\begin{gathered}
f\left(a\left(T_{1}, \ldots, T_{i} \amalg T_{i}^{\prime}, \ldots, T_{m}\right), b\left(T_{m+1}, \ldots, T_{m+n}\right)\right) \\
\quad \cong f\left(a\left(T_{1}, \ldots, T_{i}, \ldots, T_{m}\right) \oplus a\left(T_{1}, \ldots, T_{i}^{\prime}, \ldots, T_{m}\right), b\left(T_{m+1}, \ldots, T_{m+n}\right)\right) \\
\cong f\left(a\left(T_{1}, \ldots, T_{i}, \ldots, T_{m}\right), b\left(T_{m+1}, \ldots, T_{m+n}\right)\right) \\
\oplus f\left(a\left(T_{1}, \ldots, T_{i}^{\prime}, \ldots, T_{m}\right), b\left(T_{m+1}, \ldots, T_{m+n}\right)\right)
\end{gathered}
$$


and $\delta^{\prime}\left(1 \times \beta^{i}\right)$ the family of isomorphisms

$$
\begin{gathered}
f\left(a\left(T_{1}, \ldots, T_{m}\right), b\left(T_{m+1}, \ldots, T_{m+i} \amalg T_{m+i}^{\prime}, \ldots, T_{m+n}\right)\right) \\
\cong f\left(a\left(T_{1}, \ldots, T_{m}\right), b\left(T_{m+1}, \ldots, T_{m+i}, \ldots, T_{m+n}\right)\right) \oplus \\
\quad f\left(a\left(T_{1}, \ldots, T_{m}\right), b\left(T_{m+1}, \ldots, T_{m+i}^{\prime}, \ldots, T_{m+n}\right)\right)
\end{gathered}
$$

similarly defined.

One easily checks that the induced maps

$$
C_{m, n} f: \mathbb{C}_{m} U \wedge C_{n} V \rightarrow C_{m+n} W
$$

satisfy the condition of a pairing of $S_{*}$-spectra, and that $\mathbb{C}_{0,0} f$ coincides with $B f: B U \wedge B V \rightarrow B W$. Thus we see that the functor $U \mapsto C U$ is a multiplicative higher algebraic $\mathbb{K}$-theory defined on symmetric monoidal (or permutative) categories.

2. 3. May machine. May [7] has defined a functor from permutative categories to $\mathscr{I}_{*}$-prespectra by the composite

$$
U \mapsto \tilde{U} \mapsto B \tilde{U} \mapsto T B \tilde{U}
$$

where $\tilde{U}$ denotes the functor $\mathscr{F} \rightarrow$ Cat obtained by applying the Street's first construction [12] on a lax functor $\mathrm{n} \mapsto U^{n}$, and $T$ assigns to every $\mathscr{F}$-space $X$ an $\mathscr{I}_{*}$-prespectrum $T X: V \mapsto B\left(\Sigma^{V}, \hat{G}_{V}, X\right)$. Let us denote by $\mathbb{M} U$ the associated $S_{*}$-spectrum $\left\{\mathbb{R}_{n} U=T B \tilde{U}\left(\mathbb{R}^{n}\right) \mid n \geqq 0\right\}$.

The canonical inclusion $U \rightarrow \tilde{U}(\mathbb{1})$ induces a natural map $\lambda: B U \rightarrow$ $B \tilde{U}(\mathbb{1}) \rightarrow T B \tilde{U}(\{0\})=\mathbb{M}_{0} U$ such that the composite $B U \rightarrow \Omega^{\infty} \mathbb{M} \mathbb{L}_{\infty} U$ is a group completion.

By Theorems 2.1 and 6.2 of [7] we see that pairings $f:(U, V) \rightarrow W$ of permutative categories functorially determine pairings $\mathbb{M} f:(\mathbb{M} U, \mathbb{R} V)$ $\rightarrow \mathbb{M} W$ of $S_{*}$-spectra, and it is easy to see that the following square commutes ;

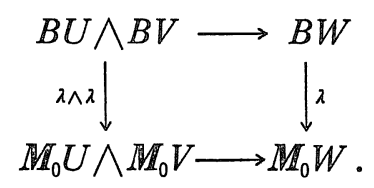

Therefore the functor $U \mapsto \mathbb{M} U$ also becomes a multiplicative higher algebraic $\mathbb{K}$-theory defined on permutative categories.

Remark. Unfortunately, Segal machine applied to the $\Gamma$-space $B \hat{B} U=B \bar{U}$ does not provide an $S_{*}$-spectrum. In fact, Segal-Woolfson 
approach to constructing pairings of spectra is much more complicated than that described here. (Compare [16] and [9].)

\section{§ 3. $S_{*}$-Bispectra and Their Pairings}

A $C W$-bispectrum is a family $X=\left\{X_{n, q} \mid n \geqq 0, q \geqq 0\right\}$ of $C W$-complexes $X_{n, q}$ equipped with cellular embeddings

$$
\begin{array}{ll}
X_{n, q} \wedge S^{1} \rightarrow X_{n+1, q}, & (x, s) \mapsto[x, s] \\
X_{n, q} \wedge S^{1} \rightarrow X_{n, q+1}, & (x, s) \mapsto[x, s]^{\prime}
\end{array}
$$

such that

$$
[[x, s], t]^{\prime}=\left[[x, t]^{\prime}, s\right]
$$

holds for all $(x, s, t) \in X_{n, q} \wedge S^{1} \wedge S^{1}$. Every $C W$-bispectrum $X$ determines (and is uniquely determined by) $C W$-spectra

$$
X_{n *}=\left\{X_{n, q} \mid q \geqq 0\right\}, X_{* q}=\left\{X_{n, q} \mid n \geqq 0\right\}
$$

and functions of spectra

$$
X_{n *} \wedge S^{1} \longrightarrow X_{n+1 *}, X_{* q} \wedge S^{1} \longrightarrow X_{* q+1} \text {. }
$$

For any bispectrum $X$ we define two diagonal spectra $D X$ and $D^{\prime} X$ as follows (cf. [8]):

$$
D_{n} X=\Omega^{n} X_{n, n}=D_{n}^{\prime} X
$$

and the structure maps $\delta: D_{n} X \wedge S^{1} \rightarrow D_{n+1} X$ and $\delta^{\prime}: D_{n}^{\prime} X \wedge S^{1} \rightarrow D_{n+1}^{\prime} X$ are given by

$$
\begin{aligned}
& \delta(x, s)\left(t_{1}, \ldots, t_{n}, t_{n+1}\right)=\left[\left[x\left(t_{1}, \ldots, t_{n}\right), t_{n+1}\right], s\right]^{\prime} \\
& \delta^{\prime}(x, s)\left(t_{1}, \ldots, t_{n}, t_{n+1}\right)=\left[\left[x\left(t_{1}, \ldots, t_{n}\right), t_{n+1}\right]^{\prime}, s\right]
\end{aligned}
$$

for all $(x, s) \in \Omega^{n} X_{n, n} \wedge S^{1}$ and $\left(t_{1}, \ldots, t_{n}, t_{n+1}\right) \in S^{n+1}$. Then there are maps of spectra (natural in $H \mathscr{S}$ ) $e: X_{0 *} \rightarrow D X$ given by

$$
e_{q} x\left(t_{1}, \ldots, t_{q}\right)=\left[x, t_{1}, \ldots, t_{q}\right]
$$

for every $x \in X_{0, q} ; e^{\prime}: X_{* 0} \rightarrow D^{\prime} X$ given by

$$
e_{n}^{\prime} y\left(t_{1}, \ldots, t_{n}\right)=\left[y, t_{1}, \ldots, t_{n}\right]^{\prime}
$$

for every $y \in X_{n, 0}$; and $c=d^{\prime} \rho^{-1}: D X \stackrel{\simeq}{\longrightarrow} D^{\prime} X$ where $\rho^{-1}$ is the homotopy inverse of the canonical map $\rho: T D X \rightarrow D X$ and $d^{\prime}: T D X \rightarrow D^{\prime} X$ is induced from the preternatural weak map $d: D X \rightarrow D^{\prime} X$;

$$
d_{n} x\left(t_{1}, \ldots, t_{n}\right)=x\left(-t_{1}, \ldots,-t_{n}\right)
$$


for every $x \in D_{n} X$ and $\left(t_{1}, \ldots, t_{n}\right) \in S^{n}$. (Compare [8, Appendix A].) Explicitly we fix a homotopy $k_{t}=\left(f_{t}, g_{t}\right): S^{2} \rightarrow S^{2}$ such that $k_{0}(u, v)=$ $(u, v), k_{1}(u, v)=(v,-u)$ and use the following homotopies $h_{n}: d_{n+1} \delta_{n}$ $\simeq \delta_{n}^{\prime}\left(d_{n} \wedge 1\right)$ to define $d^{\prime}$;

$$
\left(h_{n}\right)_{t}(x, s)\left(t_{1}, \ldots, t_{n}, t_{n+1}\right)=d_{n+1} \delta_{n}\left(x, f_{t}\left(s, t_{n+1}\right)\right)\left(t_{1}, \ldots, t_{n}, g_{t}\left(s, t_{n+1}\right)\right)
$$

Definition 3.1. A $C W$-bispectrum $X$ is called an $S_{*}$-bispectrum if every $X_{m *}$ and $X_{* p}$ have a structure of an $S_{*}$-spectrum, and if $X_{m *} \wedge S^{1} \rightarrow X_{m+1 *}$ and $X_{* p} \wedge S^{1} \rightarrow X_{* p+1}$ are functions of $S_{*}$-spectra. (Thus each $X_{n, q}$ has an $S_{n} \times S_{q}$-action such that

$$
\left(\sigma+\sigma^{\prime}, \tau+\tau^{\prime}\right)[[x, s], t]^{\prime}=\left[\left[(\sigma, \tau) x, \sigma^{\prime} s\right], \tau^{\prime} t\right]^{\prime}
$$

for every $\sigma \in S_{n}, \quad \tau \in S_{q}, \quad \sigma^{\prime} \in S_{k}, \quad \tau^{\prime} \in S_{r}$ and $(x, s, t) \in X_{n, q} \wedge S^{k} \wedge S^{r}$. $)$ Given $S_{*}$-bispectra $X, Y$ and $Z$, a pairing $\mu:(X, Y) \rightarrow Z$ of $S_{*}$-bispectra is a family of maps

$$
X_{m, q} \wedge Y_{n, q} \rightarrow Z_{m+n, p+q}
$$

which restricts to pairings of $S_{*}$-spectra

$$
\left(X_{m *}, Y_{n *}\right) \rightarrow Z_{m+n *} \text { and }\left(X_{* p}, Y_{* q}\right) \rightarrow Z_{* p+q} \text {. }
$$

For example, the $C W$-bispetrum $\Delta S=\left\{S^{n} \wedge S^{q} \mid n \geqq 0, q \geqq 0\right\}$ equipped with the structure maps

$$
\begin{array}{ll}
{[(x, y), s]=((x, s), y),} & {[(x, y), s]^{\prime}=(x,(y, s))} \\
& \text { for }((x, y), s) \in \Delta S_{n, q} \wedge S^{1}
\end{array}
$$

canonically has a structure of an $S_{*}$-bispectrum, and for every $S_{*-}$ bispectrum $X$ we have a natural pairing $\tilde{\varepsilon}:(X, \Delta S) \rightarrow X$ of $S_{*}$-bispectra;

$$
\begin{aligned}
& X_{m, p} \wedge\left(S^{n} \wedge S^{q}\right) \longrightarrow X_{m+n, p+q} \text { 。 } \\
& \Psi \quad \Psi \\
& (x,(s, t)) \longmapsto[[x, s], t]^{\prime}
\end{aligned}
$$

It is easy to see that if $X$ is an $S_{*}$-bispectrum then both $D X$ and $D^{\prime} X$ are $S_{*}$-spectra with the $S_{n}$-action

$$
\left(\sigma, S^{n} \stackrel{x}{\longrightarrow} X_{n, n}\right) \longmapsto\left(S^{n} \stackrel{\sigma^{-1}}{\longrightarrow} S^{n} \stackrel{x}{\longrightarrow} X_{n, n} \stackrel{(\sigma, \sigma)}{\longrightarrow} X_{n, n}\right)
$$

on each $D_{n} X=\Omega^{n} X_{n, n}=D_{n}^{\prime} X$, and also that $e: X_{0 *} \rightarrow D X$ and $e^{\prime}: X_{* 0} \rightarrow$ $D^{\prime} X$ are functions of $S_{*}$-spectra. (However $c: D X \rightarrow D^{\prime} X$ is not a function of $S_{*}$-spectra.) Now let $\mu:(X, Y) \rightarrow Z$ be a pairing of $S_{*^{-}}$ bispectra. Then the diagram 


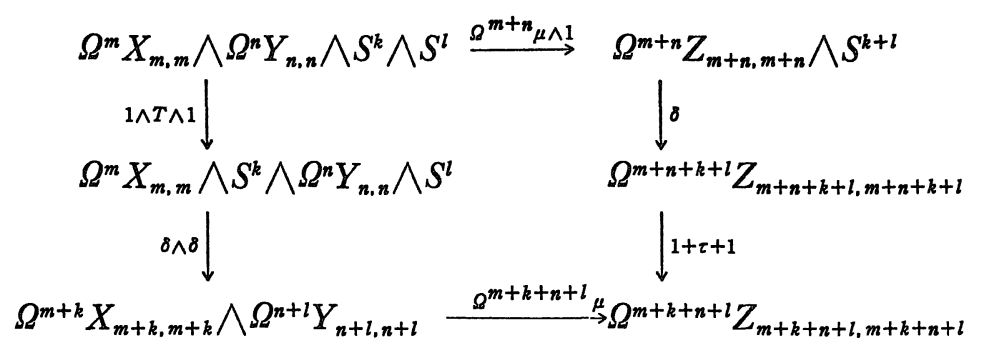

commutes. Hence the maps $\Omega^{m+n} \mu: \Omega^{m} X_{m, m} \wedge \Omega^{n} Y_{n, n} \rightarrow \Omega^{m+n} Z_{m+n, m+n}$ define a pairing $D \mu:(D X, D Y) \rightarrow D Z$ of $S_{*}$-spectra, and similarly $D^{\prime} \mu$ : $\left(D^{\prime} X, D^{\prime} Y\right) \rightarrow D^{\prime} Z$. The following proposition is a multiplicative version of the up and across theorem (cf. [2] and [8]).

Proposition 3.2. Let $\mu:(X, Y) \rightarrow Z$ be a pairing of $S_{*}$-bispectra. Then the following diagram commutes in the stable category;

(*)

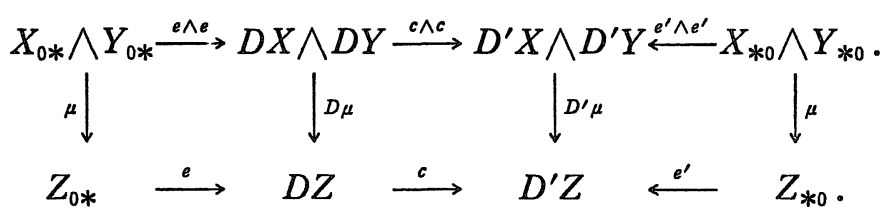

Proof. For every $m$ and $n$, we have a commutative diagram

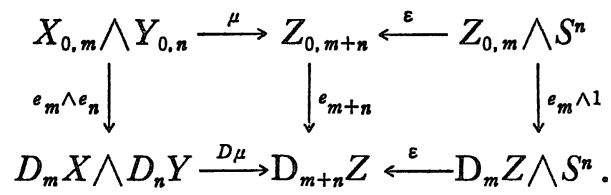

Noting that $e$ is a map of $S_{*}$-spectra (and hence induces We: $W Z_{0 *} \rightarrow W D Z$ ), we can easily show that the left-hand square in (*) commutes.

Quite similarly we can prove the commutativity of the right-hand square in (*).

Finally, to prove the commutativity of the middle square, let us take a partition of $N=B \cup C$, and denote $n^{\prime}=\beta(n), n^{\prime \prime}=\gamma(n)$ for every $n \in N$. Then there is a canonical inclusion $T\left(D X \wedge_{B C} D Y\right) \rightarrow T D X \wedge_{B C}$ $T D Y$ which sends $[k]_{+} \wedge\left(D_{k^{\prime}} X \wedge D_{k^{\prime \prime}} Y\right) \wedge S^{n-k} \subset T_{n}\left(D X \bigwedge_{B C} D Y\right)$ to $\left(\left[k^{\prime}\right]_{+} \wedge D_{k^{\prime}} X \wedge S^{n^{\prime}-k^{\prime}}\right) \wedge\left(\left[k^{\prime \prime}\right]_{+} \wedge D_{k^{\prime \prime}} Y \wedge S^{n^{\prime \prime}-k^{\prime \prime}}\right) \subset T_{n^{\prime}} D X \wedge T_{n^{\prime \prime}} D Y$, and we have a commutative diagram 


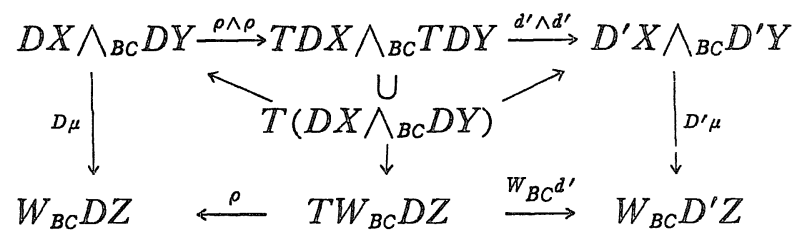

in which $W_{B C} d^{\prime}$ is defined by using the natural homotopies $d_{n+1}\left(W_{B C} \delta\right)_{n} \simeq\left(W_{B C} \delta^{\prime}\right)_{n}\left(d_{n} \wedge 1\right)$

$$
\tilde{h}_{n}= \begin{cases}h_{n} & \text { if } n \in C \\ \sigma_{n^{\prime}, n^{\prime \prime}} h_{n}\left(1 \wedge(-1)^{n^{\prime \prime}}\right) & \text { if } n \in B .\end{cases}
$$

From this and the similar diagram with $\mu$ replaced by $\tilde{\varepsilon}:(Z, \Delta S) \rightarrow Z$, we see that

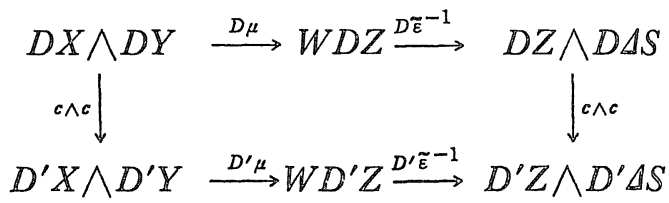

commutes in the stable category. Because the composite $T D Z \rightarrow$ $D Z \wedge D \Delta S \rightarrow W D Z$ (resp. $T D^{\prime} Z \rightarrow D^{\prime} Z \wedge D^{\prime} \Delta S \rightarrow W D^{\prime} Z$ ) coincides with $T D Z \rightarrow D Z \wedge S^{0} \rightarrow W D Z$ (resp. $T D^{\prime} Z \rightarrow D^{\prime} Z \wedge S^{0} \rightarrow W D^{\prime} Z$ ), we conclude that the middle square in $(*)$ commutes.

\section{\$4. Proofs of the Theorems}

4. 1. Proof of Theorem $\mathbb{A}$. Let $U$ be a permutative category. Then we have a bispectrum $\mathbb{X} U=\left\{\mathbb{X}_{n, q} U \mid n \geqq 0, q \geqq 0\right\}$ defined as follows:

$\mathbb{X}_{n, q} U$ is the geometric realization of the $\Gamma^{q}$-space

$$
\mathbb{E}_{n} \hat{B}^{q} U:\left(\mathbb{1}_{1}, \ldots, \mathbb{P}_{q}\right) \longmapsto \mathbb{E}_{n} \hat{B}^{q} U\left(\mathbb{1}_{1}, \ldots, \mathbb{T}_{q}\right) \text {; }
$$

the structure maps $X_{n, q} U \wedge S^{1} \rightarrow \mathbb{X}_{n+1, q} U$ and $\mathbb{X}_{n, q} U \wedge S^{1} \rightarrow \mathbb{X}_{n, q+1} U$ are given by the evident maps

$$
\begin{aligned}
& \left|\mathbb{E}_{n} \hat{B}^{q} U\right| \wedge S^{1}=\left|\mathbb{E}_{n} \hat{B}^{q} U \wedge S^{1}\right| \rightarrow\left|\mathbb{E}_{n+1} \hat{B}^{q} U\right| \\
& \left|\mathbb{E}_{n} \hat{B}^{q} U\right| \wedge S^{1}=\left|\mathbb{E}_{n} \hat{B}^{q+1} U(\circ \circ, \mathbb{1})\right| \wedge S^{1} \rightarrow\left|\mathbb{E}_{n} \hat{B}^{q+1} U\right|
\end{aligned}
$$

respectively.

By the definition we have $\mathbb{X}_{*_{0}} U=\mathbb{E} U$ and there is a natural map $\mathbb{C} U \rightarrow \mathbb{X}_{0 *} U$ given by 


$$
C_{n} U=\left|B \hat{B}^{n} U\right| \stackrel{|\lambda|}{\longrightarrow}\left|\boldsymbol{E}_{0} \hat{B}^{n} U\right|=X_{0, n} U \text { 。 }
$$

Because $\left|\boldsymbol{E}_{n} \hat{B}^{q} U\right|$ is connected when $n \geqq 1$, the canonical map $\boldsymbol{X}_{n, q} U$ $\rightarrow \Omega X_{n, q+1} U$ is a homotopy equivalence for all $n \geqq 1$. Hence $e^{\prime}: \mathbb{E} U \rightarrow$ $D^{\prime} \boldsymbol{X} U$ becomes an equivalence. Moreover, because $B() \rightarrow \Omega^{\circ} \boldsymbol{E}_{\infty}(\quad)$ and $B(\quad) \rightarrow \Omega^{\circ} C_{\infty}(\quad)$ are group completions, the composite $k: C U \rightarrow$ $\boldsymbol{X}_{0 *} U \stackrel{e}{\longrightarrow} D X U$ also becomes an equivalence. Thus we have an equivalence

natural in $U$.

$$
\gamma=k^{-1} c^{-1} e^{\prime}: \mathbb{E} U \rightarrow C U
$$

Now suppose that $\boldsymbol{E}$ is a multiplicative higher algebraic $\mathrm{K}$-theory. Then $X U$ becomes an $S_{*}$-bispectrum with an $S_{n} \times S_{q}$-action on $X_{n, q} U$ induced from the $S_{n}$-action on $\mathbb{E}_{n}$ and the $S_{q}$-action on $\hat{B}^{q}$.

Let $f: U \times V \rightarrow W$ be a pairing of permutative categories. Then we have a natural pairing $\boldsymbol{X} f:(\boldsymbol{X} U, \boldsymbol{X} V) \rightarrow X W$ of $S_{*}$-bispectra consisting of the maps $X_{m, p} U \wedge X_{n, q} V \rightarrow X_{m+n, p+q} W$ induced by the map of $\Gamma^{p+q_{-} \text {spaces }}$

$$
\begin{aligned}
\mathbb{E}_{m, n} f_{p, q}: \boldsymbol{E}_{m} \hat{B}^{p} U\left(\mathbb{r}_{1}, \ldots, \mathbb{r}_{p}\right) \wedge \boldsymbol{E}_{n} \hat{B}^{q} V\left(\mathbb{r}_{p+1}, \ldots, \mathbf{r}_{p+q}\right) \\
\longrightarrow \mathbb{E}_{m+n} \hat{B}^{p+q} W\left(\mathbf{r}_{1}, \ldots, \mathbf{r}_{p}, \mathbf{r}_{p+1}, \ldots, \mathbf{r}_{p+q}\right) .
\end{aligned}
$$

By Proposition 3.2 and by the multiplicativity of $\lambda: B() \rightarrow E_{0}()$, we see that the following diagram commutes in $H \mathscr{S}$;

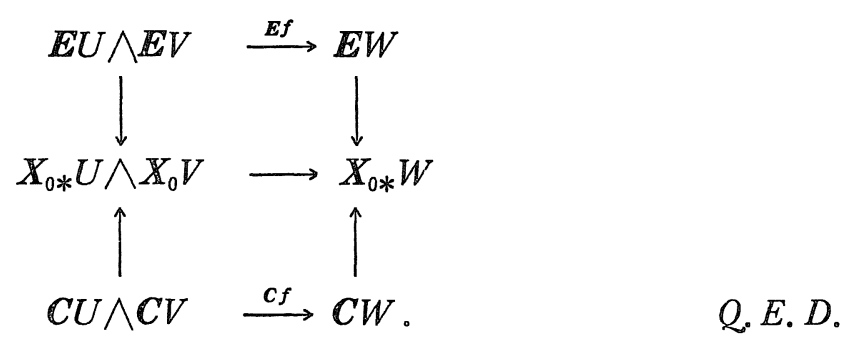

4. 2. Proof of Theorem $\mathbf{B}_{\text {。 }}$ Let $U$ be an exact category。 We first define an equivalence $\eta: \Omega C Q U \rightarrow K U$. By [10, Lemma 4.2] every $\hat{B}^{q} U\left(\mathbf{r}_{1}, \ldots, \mathbf{r}_{q}\right)$ has a natural structure of an exact category. Hence we can define an $S_{*}$-bispectrum $X U$ by

$$
\boldsymbol{X}_{n, q} U=\left|\boldsymbol{K}_{n} \hat{B}^{q} U\right|=\left|\left(\mathbf{r}_{1}, \ldots, \mathbf{r}_{q}\right) \longmapsto B Q^{n}\left(\hat{B}^{q} U\left(\mathbf{r}_{1}, \ldots, \mathbf{r}_{q}\right)\right)^{[n]}\right| .
$$

Clearly $\mathbb{X}_{*_{0}} U=\mathbb{K} U$, and by [10, Corollary 4.5] we have

$$
\begin{aligned}
X_{1 *} U & =\left\{\left|B Q \hat{B}^{n} U\right| \mid n \geqq 0\right\} \\
& =\left\{\left|B \hat{B}^{n} Q U\right| \mid n \geqq 0\right\}=C Q U .
\end{aligned}
$$


Now let us endow $\mathbb{X}^{\prime} U=\left\{\Omega X_{1+n, q} U \mid n \geqq 0, q \geqq 0\right\}$ with the structure of an $S_{*}$-bispectrum such that the maps

$$
\mathbb{X}_{n, q} U \longrightarrow \Omega X_{1+n, q} U, x \mapsto\left(s \mapsto \sigma_{0, n}[x, s]\right)
$$

give rise to a function $\phi: X U \rightarrow \mathbb{X}^{\prime} U$ of $S_{*}$-bispectra. Then $\mathbb{X}_{0 *}^{\prime} U=$ $\Omega \mathbb{X}_{1 *} U=\Omega C Q U$ and it is easy to see that the maps $e: \mathbb{X}_{0 *}^{\prime} U \rightarrow D \mathbb{X}^{\prime} U$, $e^{\prime}: \mathbb{X}_{* 0}^{\prime} U \rightarrow D^{\prime} \mathbb{X}^{\prime} U$ and $\phi: \mathbb{X}_{* 0} U \rightarrow \mathbb{X}_{* 0}^{\prime} U$ are homotopy equivalences. Thus we have a natural equivalence $\eta: \Omega C Q U \rightarrow \mathbb{K} U$ defined as the composite

$$
X_{0 *}^{\prime} U \stackrel{e}{\longrightarrow} D X^{\prime} U \stackrel{c}{\longrightarrow} D^{\prime} X^{\prime} U \stackrel{e^{\prime-1}}{\longrightarrow} X_{* 0}^{\prime} U \stackrel{\psi^{-1}}{\longrightarrow} X_{*_{0}} U
$$

Next consider the natural sequence of spectra

$$
\mathrm{CIs} U \longrightarrow \mathrm{CLU} \longrightarrow \mathrm{CQU}
$$

associated with the sequence of symmetric monoidal categories Is $U \rightarrow$ $L U \rightarrow Q U$ (cf. $[14, \S 9]$ ). As in the proof of [11, Theorem 3.1], there are adjunctions

$$
\hat{B}^{n} L U\left(\mathbb{P}_{1}, \ldots, \mathbb{r}_{n}\right) \underset{h}{\stackrel{t}{\rightleftarrows}} \hat{B}^{n} J U\left(\mathbb{P}_{1}, \ldots, \mathbb{Z}_{n}\right) \longleftrightarrow 0
$$

natural in both $U$ and $\left(\mathbb{r}_{1}, \ldots, \mathbb{r}_{n}\right)$. Therefore we have a null homotopy on every $\mathbb{C}_{n} L U=\left|B \hat{B}^{n} L U\right|$ which is compatible with the structure maps of $C L U$, and is natural in $U$. Since the composite $\mathbb{C}_{n}$ Is $U \rightarrow \mathbb{C}_{n} Q U$ is the constant map, we have a natural map $\nu: \mathbb{C I s} U \rightarrow$ $\Omega C Q U$, and hence the composite

$$
\kappa=\eta \nu: \mathbb{C I s} U \longrightarrow \mathbb{R} U \text { 。 }
$$

We now prove that $\kappa$ is multiplicative. Let $f: U \times V \rightarrow W$ be a biexact functor. Then, as in the proof of Theorem $A$, there is a natural pairing $\mathbb{X} f:(\mathbb{X} U, X V) \rightarrow \mathbb{X} W$ of $S_{*}$-bispectra such that $\mathbb{X}_{m, p} U \wedge$ $\mathbb{X}_{n, q} V \rightarrow \mathbb{X}_{m+n, p+q} W$ is induced from the $(m+n)$-fold functors

$$
\begin{gathered}
Q^{m}\left(\hat{B}^{p} U\left(\mathbb{r}_{1}, \ldots, \mathbb{x}_{p}\right)\right)^{[m]} \Pi Q^{n}\left(\hat{B}^{q} V\left\{r_{p+1}, \ldots, \mathbb{r}_{p+q}\right)\right)^{[n]} \\
\longrightarrow Q^{m+n}\left(\hat{B}^{p+q} W\left(\mathbb{r}_{1}, \ldots, \mathbb{r}_{p+q}\right)\right)^{[m+n]}
\end{gathered}
$$

associated with the biexact functor $f_{p, q}: \hat{B}^{p} U\left(\mathbb{P}_{1}, \ldots, \mathbb{P}_{p}\right) \times \hat{B}^{q} V\left(\mathbb{P}_{p+1}\right.$, $\left.\ldots, \mathbb{1}_{p+q}\right) \rightarrow \hat{B}^{p+q} W\left(\mathbb{r}_{1}, \ldots, \mathbb{r}_{p+q}\right) \quad(\mathrm{cf} .[11, \S 4])$. It is easy to see that the composite maps

$$
\Omega X_{1+m, p} U \wedge \Omega \mathbb{X}_{1+n, q} V \stackrel{\Omega^{2} X f}{\longrightarrow} \Omega^{2} \mathbb{X}_{1+m+1+n, p+q} W \longrightarrow \Omega^{2} \mathbb{X}_{2+m+n, p+q} W
$$

define a pairing of $S_{*}$-bispectra $\mathbb{X}^{\prime} f:\left(\mathbb{X}^{\prime} U, \mathbb{X}^{\prime} V\right) \rightarrow \mathbb{X}^{\prime \prime} W$ where 
$\boldsymbol{X}^{\prime \prime} W=\left\{\Omega^{2} \boldsymbol{X}_{2+n, q} W \mid n \geqq 0, q \geqq 0\right\}$ is equipped with the structure of an $S_{*}$-bispectrum evidently defined. Thus we have a commutative diagram

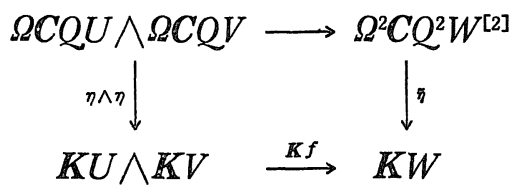

where $\Omega^{2} C Q^{2} W^{[2]}$ denotes $\mathbb{X}_{0 *}^{\prime \prime} W=\Omega^{2} \boldsymbol{X}_{2 *} W$, and $\tilde{\eta}$ the composite

$$
X_{0 *}^{\prime \prime} W \stackrel{\simeq}{\longrightarrow} X_{* 0}^{\prime \prime} W \stackrel{\simeq}{\longrightarrow} X_{* 0} W .
$$

We now define a natural map $\tilde{\Sigma}: \mathbb{C}$ Is $W \rightarrow \Omega^{2} C Q^{2} W^{[2]}$ such that the following diagram commutes in the stable category (cf. $[14,9.2]$ );

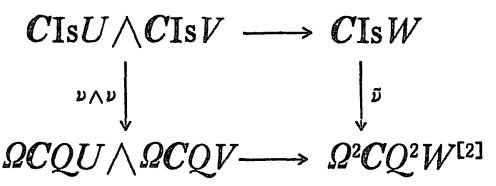

As stated in $[11, \S 3]$, any biexact functor $g: C \times D \rightarrow E$ defines a 2-fold functor $C \Pi D \rightarrow E^{[2]}$ which induces a commutative diagram

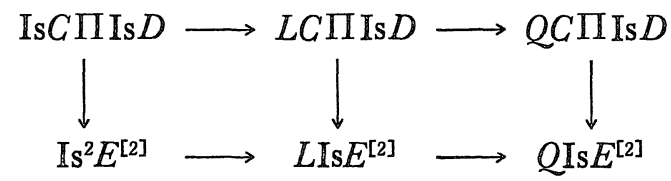

natural in $g$. (We denote $\mathrm{Is}^{2}=\mathrm{Is}_{1} \mathrm{Is}_{2}, L \mathrm{Is}=L_{1} \mathrm{Is}_{2}$ and $Q \mathrm{Is}=Q_{1} \mathrm{Is}_{2}$. Cf. [11].) Applying this construction to biexact functors

$$
f_{p, q}: \hat{B}^{p} U\left(\mathbb{r}_{1}, \ldots, \mathbb{r}_{p}\right) \times \hat{B}^{q} V\left(\mathbb{r}_{p+1}, \ldots, \mathbb{r}_{p+q}\right) \rightarrow \hat{B}^{p+q} W\left(\mathbb{r}_{1}, \ldots, \mathbb{r}_{p+q}\right),
$$

and then realizing the associated $\Gamma^{p+q}$-spaces, we have a commutative diagram

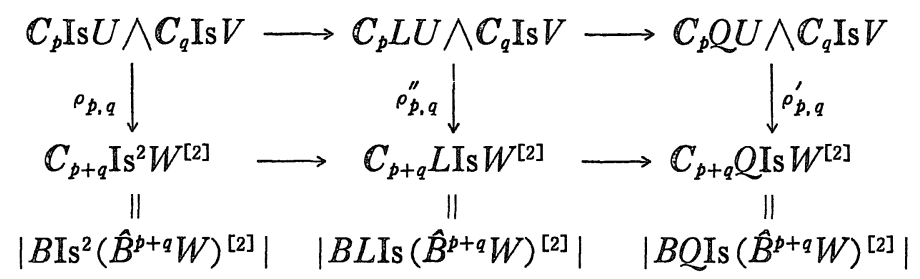

for all $p, q \geqq 0$. Since $\rho_{p, q}^{\prime \prime}$ is compatible with the null homotopies, the following diagram commutes; 


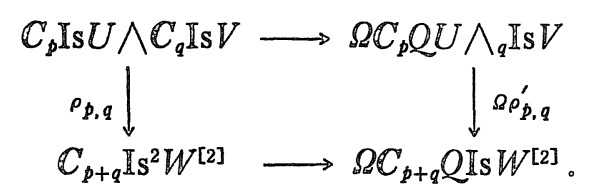

By the definition, $\left\{\rho_{p, q}\right\}$ and $\left\{\rho_{p, q}^{\prime}\right\}$ are pairings of $S_{*}$-spectra, and hence we have a diagram

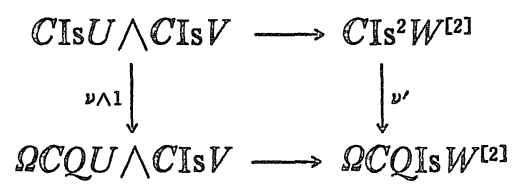

which commutes in the stable category.

Similarly we have a commutative diagram

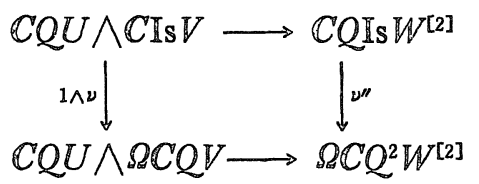

associated with the natural sequence

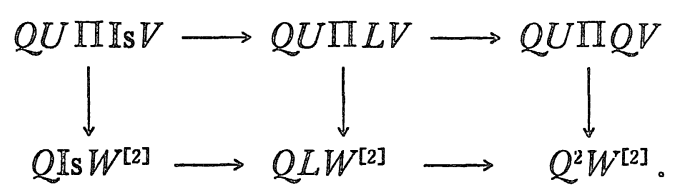

By $[14,9.2 .3]$ we see that the following diagram commutes up to natural homotopy

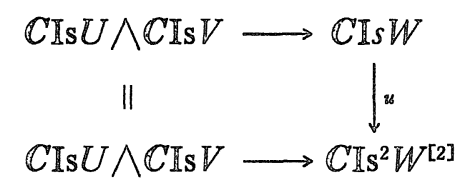

where $u_{n}: \mathbb{C}_{n} \mathrm{I} \mathrm{s} W \stackrel{\simeq}{\longrightarrow} \mathbb{C}_{n} \mathrm{Is}^{2} W^{[2]}$ denotes the canonical inclusion

$$
\left|B \mathbb{I s}^{n} W\right|=\left|B \mathbb{I s}_{1} o_{2}\left(\hat{B}^{n} W\right)^{[2]}\right| \rightarrow\left|B \mathbb{I s}^{2}\left(\hat{B}^{n} W\right)^{[2]}\right|_{\text {。 }}
$$

From (4.2), (4.3) and (4.4), we see that (4.1) commutes if we put $\tilde{\nu}=\left(\Omega \nu^{\prime \prime}\right) \nu^{\prime} u$. Moreover, from the commutative diagram

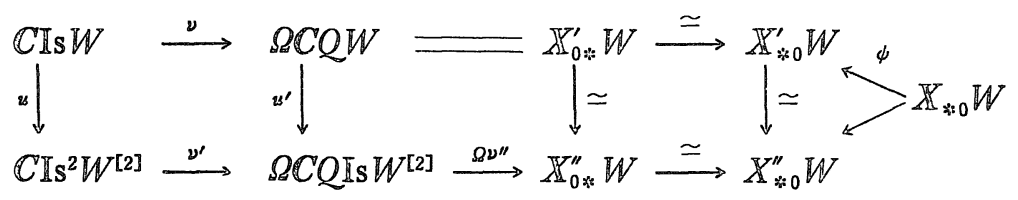


in which $u^{\prime}$ consists of the canonical inclusions $u_{n}^{\prime}:\left|B Q_{0}\left(\hat{B}^{n} W\right)^{[2]}\right| \rightarrow$ $\left|B Q I s\left(\hat{B}^{n} W\right)^{[2]}\right|$, we see that the composite $\tilde{\eta} \tilde{\nu}: C$ Is $W \rightarrow \Omega^{2} C Q^{2} W^{[2]} \rightarrow \mathbb{K} W$ coincides with $\kappa=\eta \nu$. Therefore the diagram

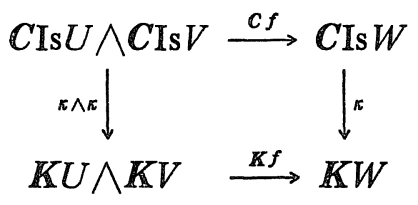

commutes in the stable category.

Q.E. D.

4. 3. Proof of Theorem C. Given a ring $R$, we define $A R$ as follows. For each $n \geqq 0$,

$$
A_{n} R=\Omega^{n} C_{n} \operatorname{Is} P\left(S^{n} R\right)
$$

where $S^{n} R=R \otimes\left(\stackrel{n}{\otimes} S Z\right.$ ) (cf. [5]); and the structure map $A_{n} R \wedge S^{1} \rightarrow$ $A_{n+1} R$ is defined as the composite

$$
\begin{aligned}
A_{n} R \wedge & S^{1} \stackrel{1 \wedge \iota}{\longrightarrow} \Omega^{n} C_{n} \operatorname{Is} P\left(S^{n} R\right) \wedge \Omega C_{1} \operatorname{Is} P(S \mathscr{Z}) \\
& \stackrel{\Omega^{n+1} C_{n, 1}^{f}}{\longrightarrow} \Omega^{n+1} C_{n+1} \operatorname{Is} P\left(S^{n+1} R\right)=A_{n+1} R
\end{aligned}
$$

where $f$ denotes the evident pairing

$$
\text { Is } P\left(S^{n} R\right) \times \operatorname{Is} P(S Z) \longrightarrow \operatorname{Is} P\left(S^{n} R \otimes S Z\right)=\operatorname{Is} P\left(S^{n+1} R\right),
$$

and

$$
\iota: S^{1} \longrightarrow B I s P(S Z) \subset \Omega C_{1} \text { Is } P(S Z)
$$

the cellular inclusion corresponding to the ' 1 -cell'

$$
\left[\begin{array}{lllll}
0 & 0 & 0 & \cdot & \cdot \\
1 & 0 & 0 & \cdot & \cdot \\
0 & 1 & 0 & \cdot & \cdot \\
0 & 0 & 1 & \cdot & \cdot \\
\cdot & \cdot & \cdot & \cdot & .
\end{array}\right] \in G L_{1} S \mathbb{Z} .
$$

Note that e represents a generator of $K_{1} S \boldsymbol{Z}=K_{0} \mathbb{Z}=\mathbb{Z}$ (cf. [5]).

Using the standard $S_{n}$-action on $\otimes^{\pi} S Z$, we define an $S_{n}$-action on $A_{n} R$ by

$$
\begin{aligned}
& \left(\tau, S^{n} \stackrel{x}{\longrightarrow} C_{n} \operatorname{Is} P\left(S^{n} R\right)\right) \mapsto\left(S^{n} \stackrel{\tau^{-1}}{\longrightarrow} S^{n} \stackrel{x}{\longrightarrow} C_{n} \operatorname{Is} P(R \otimes(\stackrel{n}{\otimes} S \mathbb{Z})\right. \\
& \left.\stackrel{{ }^{\imath} C_{n}^{\mathrm{Is} P(1 \otimes r)} \longrightarrow}{\longrightarrow} C_{n} \mathrm{Is} P\left(R \otimes\left(\bigotimes^{n} S \mathbb{Z}\right)\right)\right) .
\end{aligned}
$$


Then it is easy to see that $\mathbb{A} R$ becomes an $S_{*}$-spectrum with respect to this action.

Given rings $R$ and $R^{\prime}$ we have a natural pairing

$$
\text { Is } P\left(S^{m} R\right) \times \operatorname{Is} P\left(S^{n} R^{\prime}\right) \rightarrow \operatorname{Is} P\left(S^{m} R \otimes S^{n} R^{\prime}\right) \cong \operatorname{Is} P\left(S^{m+n}\left(R \otimes R^{\prime}\right)\right)
$$

of symmetric monoidal categories, and this in turn induces a map

$$
\begin{aligned}
\mathbb{A}_{m} R \wedge \mathbb{A}_{n} R^{\prime} & =\Omega^{m} \mathbb{C}_{m} \operatorname{Is} P\left(S^{m} R\right) \wedge \Omega^{n} \mathbb{C}_{n} \operatorname{Is} P\left(S^{n} R^{\prime}\right) \\
& \longrightarrow \Omega^{m+n} C_{m+n} \operatorname{Is} P\left(S^{m+n}\left(R \otimes R^{\prime}\right)\right)=A_{m+n}\left(R \otimes R^{\prime}\right) .
\end{aligned}
$$

Thus we have a natural pairing

$$
\mu:\left(\mathbb{A} R, A R^{\prime}\right) \longrightarrow \mathbb{A}\left(R \otimes R^{\prime}\right)
$$

of $S_{*}$-spectra.

If $n \geqq 1$, then the canonical inclusion $f_{n}: B \operatorname{Is} P\left(S^{n} R\right) \rightarrow \Omega^{n} C_{n} \mathbb{I s} P\left(S^{n} R\right)$ is a group completion, and hence there is a homotopy equivalence

$$
A_{n} R \simeq K_{0} S^{n} R \times B G L S^{n} R^{+}=\mathbb{G} W_{n} R \text { 。 }
$$

By the definition we see that the square

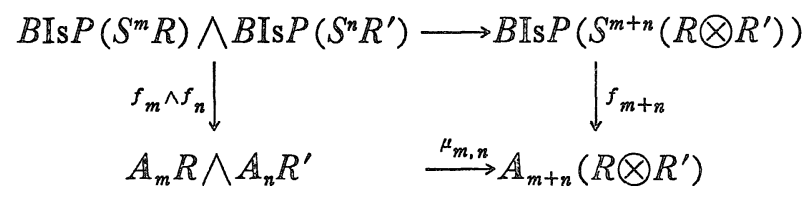

commutes, and that the structure map $\mathbb{A}_{n} R \wedge S^{1} \rightarrow \mathbb{A}_{n+1} R$ coincides with the composite $\mu_{n, 1}(1 \wedge \ell)$. Hence the conditions (2) and (3) hold.

We now define a $C W$-bispectrum $X R$ as follows.

$$
\mathbb{X}_{n, q} R=\Omega^{q} \mathbb{C}_{n+q} \operatorname{Is} P\left(S^{q} R\right) ;
$$

and the structure maps are given by

and

$$
\begin{aligned}
\mathbb{X}_{n, q} R \wedge S^{1} & =\Omega^{q} C_{n+q} \operatorname{Is} P\left(S^{q} R\right) \wedge S^{1} \\
& \longrightarrow \Omega^{q} C_{n+q+1} \operatorname{Is} P\left(S^{q} R\right) \\
& \stackrel{\Omega^{q} q_{n, q}}{\longrightarrow} \Omega^{q} C_{n+1+q} \operatorname{Is} P\left(S^{q} R\right)=\mathbb{X}_{n+1, q} R
\end{aligned}
$$

$$
\begin{aligned}
& \mathbb{X}_{n, q} R \wedge S^{1} \stackrel{1 \wedge \epsilon}{\longrightarrow} \Omega^{q} C_{n+q} \operatorname{IS} P\left(S^{q} R\right) \wedge \Omega \mathbb{C}_{1} \operatorname{Is}(S \mathbb{Z}) \\
& \longrightarrow \Omega^{q+1} C_{n+q+1} \operatorname{Is} P\left(S^{q+1} R\right)=\mathbb{X}_{n, q+1} R .
\end{aligned}
$$

It is easy to see that $\mathbb{X} R$ becomes an $S_{*}$-bispectrum if each $\mathbb{X}_{n, q} R$ is endowed with the $S_{n} \times S_{q}$-action

$$
\begin{aligned}
& \left((\sigma, \tau), S^{q} \stackrel{x}{\longrightarrow} \mathbb{C}_{n+q} \mathbb{I S} P\left(S^{q} R\right)\right) \longmapsto\left(S^{q} \stackrel{\tau^{-1}}{\longrightarrow} S^{q} \stackrel{x}{\longrightarrow} \mathbb{C}_{n+q} \operatorname{Is} P\left(S^{q} R\right)\right. \\
& \left.\stackrel{(\sigma+\tau) c_{n+q} \operatorname{Is} P(1 \otimes \tau)}{\longrightarrow} \mathbb{C}_{n+q} \operatorname{Is} P\left(S^{q} R\right)\right) \text {. }
\end{aligned}
$$


Since $e: X_{0 *}=A R \rightarrow D X R$ is a homotopy equivalence, we have a natural map

$$
\alpha: C \operatorname{Is} P(R)=X_{* 0} R \longrightarrow A R
$$

(natural in $H \mathscr{S}$ ).

Finally the maps $X_{m, p} R \wedge X_{n, q} R^{\prime} \rightarrow X_{m+n, p+q}\left(R \otimes R^{\prime}\right)$ defined as the composite

$$
\begin{aligned}
& \Omega^{p} C_{m+p} \operatorname{Is} P\left(S^{p} R\right) \wedge \Omega^{q} C_{n+q} \operatorname{Is} P\left(S^{q} R^{\prime}\right) \\
& \longrightarrow \Omega^{p+q} C_{m+p+n+q} \operatorname{Is} P\left(S^{p+q}\left(R \otimes R^{\prime}\right)\right) \\
& \stackrel{\Omega^{p+q}(1+\tau+1)}{\longrightarrow} \Omega^{p+q} C_{m+n+p+q} \operatorname{Is} P\left(S^{p+q}\left(R \otimes R^{\prime}\right)\right)
\end{aligned}
$$

determine a natural pairing $\left(\boldsymbol{X} R, \boldsymbol{X} R^{\prime}\right) \rightarrow \boldsymbol{X}\left(R \otimes R^{\prime}\right)$ of $S_{*}$-bispectra. Hence $\alpha$ becomes a multiplicative natural transformation.

Q. E. D.

\section{References}

[1] Adams, J. F., Stable homotopy and generalised homology, The University of Chicago Press, 1974.

[2] Fiedorowitz, Z., A note on the spectra of algebraic K-theory, Topology, 16 (1977), 417-421.

[3] Gersten, S., On the spectrum of algebraic K-theory, Bull. Amer. Math. Soc., 78 (1972), 216-219.

[4] Grayson, D., Higher algebraic K-theory II (after D. Quillen), in Algebraic K-theory: Evanston 1976, Lecture Notes in Math., 551, Springer, 1977.

[5] Loday, J.-L., K-théory algébrique et representations de groupes, Ann. Scient. Éc. Norm. Sup., 9 (1976), 309-377.

[6] May, J. P., The spectra associated to permutative categories, Topology, 17 (1978), 225 $-228$.

[7] 299-346.

[8] May, J. P. and Thomason, R., The uniqueness of infinite loop space machines, Topo$\log y, 17$ (1978), 205-224.

[9] Segal, G., Categories and cohomology theories, Topology, 13 (1974), 293-312.

[10] Shimada, N. and Shimakawa, K., Delooping symmetric monoidal categories, Hiroshima Math.J., 9 (1979), 627-645.

[11] Shimakawa, K., Multiple categories and algebraic K-theory, J. Pure and Appl. Algebra, 41 (1986), 285-304.

[12] Street, $\mathrm{R}_{\circ}$, Two constructions on lax functors, Cahiers de Topologie et Géometrie Differentielle, 13 (1972), 217-264.

[13] Wagoner, J., Delooping classifying spaces in algebraic K-theory, Topology, 11 (1972), 349-370.

[14] Waldhausen, F., Algebraic K-theory of generalized free products, Ann. of Math., 108 (1978), 135-256.

[15] Weibel, C., A Survey of products in algebraic K-theory, in Algebraic K-theory: Evanston 1980, Lecture Notes in Math., 854, Springer, 1981.

[16] Woolfson, R., Hyper Г-spaces and hyperspectra, Quart. J. Math., 30 (1979), 229-255. 